\title{
A Corner Potential Flow based Shape Descriptor for Object Recognition
}

\author{
Dr. M. Radhika Mani \\ Dr. Potukuchi D.M. \\ Dr. Ch. Satyanarayana
}

\begin{abstract}
Automated object recognition methods are essential for numerous applications of machine vision and pattern recognition. For an efficient object representation, a contourbased shape descriptor is designed, with a one dimensional shape signature. The present paper proposes a novel shape signature for recognizing the objects in complex plane. The proposed shape signature is applied on the contour shape representation, and then the description of representative shape features with the corner potential flow measure followed by the Fourier transformation. During recognition process, the Euclidean distance measure is evaluated to estimate the similarity score between the objects. The recital of the proposed shape descriptor has been checked with the Kimia 99 database. The experimental results are found to be robust and invariant to transformations.
\end{abstract}

Keywords-complex Plane, Potential, Sgnature, Distance Measure and Conformal Mapping.

\section{Introduction}

Recently, the applications of machine vision and pattern recognition $[1,2,3,4]$ include the object recognition techniques. Currently, object recognition plays an important role [1] in various real time applications [5,6,7]. The bottom-up object recognition methods $[8,9,10]$ focuses on the image features where as the top-down object recognition methods [11,12] focuses on the configuration of the object. Among various object recognition methods, the shape based methods are observed to be efficient [13]. Various shape based object recognition methods includes generic Fourier transform [14], convex hull [14], shock graph [15] and shape matrix [16] etc. The shape of the object can be efficiently represented by the invariant signature [17] [18] and the further described by the Fourier transformation [17]. In fluid dynamics, potential flow describes the velocity field as the gradient of a scalar function. The complex plane of the shape can be prominent for shape representation and description [19]. In the complex plane, the flow of velocity can be efficiently represented with the complex potential $[20]$.

Dr. M. Radhika Mani, Associate Professor Department of CSE, Pragati Engineering College India.

Dr. Potukuchi D.M., Professor

Department of Physics, JNT University Kakinada, Kakinada India.

Dr. Ch. Satyanarayana, Professor Department of CSE, JNT University Kakinada, Kakinada India.
Potential flow in two dimensions is simple to analyze using conformal mapping, by the use of transformations of the complex plane. The flow computation can be measured around a semi-infinite plate, around a right corner, through a corner or near a stagnation point, and around a source doublet etc. Among these the present paper computes the flow near a stagnation point and designs a Corner Potential Flow (CPF) signature for the representation of the shape of the object in the complex plane. The paper is organized in 3 -sections. Introduction to complex potential based shape representation and description methods are reported in Section-I. The details of methodology implemented presently and the relevance are detailed in section-II. Results of implementing the novel algorithm designed by the proposed approach over the standard database and their analysis with relevant discussion are presented in SectionIII.

\section{Methodology}

A novel corner based complex potential signature is proposed for shape based object recognition. The step by step details of proposed descriptor are presented in section II.A, while the section II.B discusses regarding the measures of performance.

\section{A. Design of the System}

The proposed method consists of four stages given as follows

i. Contour based shape representation of the object

ii. Computation of Corner Potential Flow Signature

iii. Fourier transformation based description

iv. Shape toning and ranking respectively.

From the Literature, it is found that the Contour based representation is prominent than the region for shape representation for object recognition. Hence, the present paper represents the contour of the shape in the initial stage followed by the Equivalent Arc Length [17] method is used for optimization. In the second stage, the represented shape information is transformed in to complex plane. The two dimensional potential flows can proficiently analyze the flow with in the shape. For this, the power-law conformal mapping is applied to the input complex plane object for estimation of potential flow at corners. The estimation of potential flow at corner is given by

$$
\phi=\mathrm{r}^{\mathrm{n}} \operatorname{Cos}(\mathrm{n} \theta)
$$

The Stream Function is estimated by

$$
\psi=\mathrm{r}^{\mathrm{n}} \operatorname{Sin}(\mathrm{n} \theta)
$$


The power $\mathrm{n}$ indicates the flow at various regions. Some of the possible values for $n$ are $\{1 / 2,2 / 3,1,2$ and -1$\}$. In the $\log$ polar transformation, for $n=1 / 2$, the flow around a semiinfinite plate, for $n=2 / 3$, the flow around a right corner, for $\mathrm{n}=1$, the uniform flow, for $\mathrm{n}=2$, the flow through a corner, or near a stagnation point, and for $n=-1$, the flow due to a source doublet is computed. The flow computation with various values of $n$ [22] is shown in Figure 1.

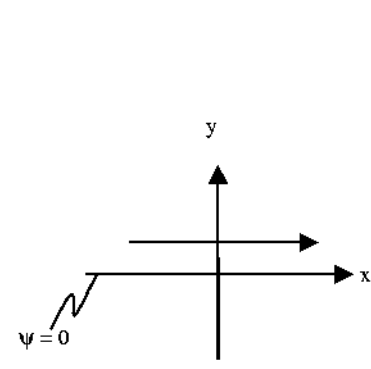

(i)

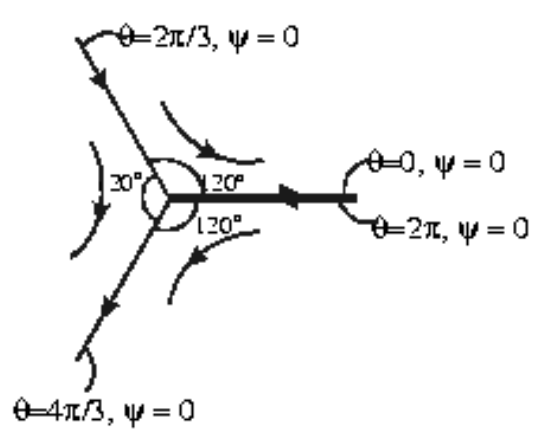

(iii)

Figure 1. Potential Flow computation with (i) $n=1$ (ii) $n=2$ and (iii) $n=3 / 2$.

The present paper considers the computes the potential flow through corner or near a stagnation point. So, the proposed Contour Potential Flow (CPF) signature considers the $\mathrm{n}$ value as 2 . The proposed CPF signature computes the potential flow at regions where the stream function value is zero. The Imaginary values of the $\mathrm{CPF}$ are in harmonic function and thus this can be used for designing the $\mathrm{CPF}$ signature.

In the third step, the description of the CPF signature is estimated with the 1-D Fourier transformation [17]. The described feature vector is found to be invariant to translation, rotation and scaling. In the wake of the fact, that the CPF signature is obtained wrt the centroid, the obtained features proved to be invariant to the translation operation. Further, the resulting finite and stipulated magnitude values of the features would be validated for the rotation invariance. The scaling invariance is achieved by dividing the feature vector with the first feature value. In the third step, the feature vector is constructed, which describes the entire shape aspect of the object. To further improve the quality of CPF signature, three global descriptors (GD) are included to the current feature vector. The GD feature vector, viz., $\{\mathrm{S}, \mathrm{C}, \mathrm{A}\}$ contains the measures of solidity, circularity and aspect ratio.

In the fourth step, the shape toning process [21] is performed with the Euclidean Distance (ED) and is estimated to test and expose to the process of shape training (i.e. during the identical shape toning process).

$$
\mathrm{ED}(\mathrm{TE}, \mathrm{TR})=\sqrt{\sum_{\mathrm{i}=1}^{\mathrm{M}}\left(\mathrm{TE}_{\mathrm{i}}-\mathrm{TR}_{\mathrm{i}}\right)^{2}}
$$

where, TE represents the test shape feature vector,

TR represents the trained shape feature vector and $\mathrm{i}$ represents the length of the feature vector.

Inclusion of GD is effectively carried out through the computation of relative distance measure given by:

$$
\begin{array}{r}
\mathrm{D}(\mathrm{TE}, \mathrm{TR})=\mathrm{ED}(\mathrm{TE}, \mathrm{TR})+\frac{\mathrm{D}_{\mathrm{X}}(\mathrm{TE}, \mathrm{TR})}{3} \\
\mathrm{D}_{\mathrm{X}}(\mathrm{TE}, \mathrm{TR})=\sum_{\mathrm{X}} \frac{\left|\mathrm{X}^{\mathrm{TE}}-\mathrm{X}^{\mathrm{TR}}\right|}{\max \left(\mathrm{X}^{\mathrm{TR}}\right)}
\end{array}
$$

where, $\mathrm{ED}(\mathrm{TE}, \mathrm{TR})$ represents the ED between the test and trained shapes,

DX(TE,TR) represents the Global distance between the test and trained shapes,

$X$ represents the GD vector $\{S, C, A R\}$,

XTE represents the GD feature of the test shape and XTR represents the GD feature of the trained shape.

The specified data of distance measurement i.e. the so computed distances are further rearranged in ascending order and are assigned with specific ranks. In turn, the system is enabled to recognize top ranked images.

\section{B. Performance Indicators}

The performance of various object recognition schemes reported so far employ different measures [17]. Among them, precision and recall are considered as important measures, while they quantify the similarity measurement. Precision (P) and Recall (R) are defined by;

$$
\begin{gathered}
\mathrm{P}=\mathrm{x} / \mathrm{y} \\
\mathrm{R}=\mathrm{x} / \text { groupsize }
\end{gathered}
$$

where,

$$
\begin{aligned}
& \mathrm{x} \text { denotes the true recognition results, } \\
& \mathrm{y} \text { denotes the total recognized result and } \\
& \text { groupsize denotes the maximum true recognition } \\
& \text { result. }
\end{aligned}
$$

The Average Precision value for each recall is computed. This value is affirmatively grouped as two categories viz., Low Recall (LR), High Recall (HR). The Average Precision for Low Recall (APLR) denotes the average precision for recalls less than equal to 50. In contrast, the Average Precision for High Recall (APHR) represents the average precision for recalls greater than 50. The proposed CPF+GD signature is compared with four standard descriptors viz., Angular Radial Transform Descriptor (ARTD) [17], 
Moment Invariant Descriptor (MID) [17], Zernike Moment Descriptor (ZMD) [17] and Curvature-Scale-SpaceDescriptor (CSSD) [17].

\section{Results and Discussions}

Due to the varied set of object shapes, the Kimia 99 database is used for evaluating the performance of the proposed Corner Potential Flow descriptor as discussed in section II-A. The results of proposed method and various other standard methods are presented in the following subsection-III.A. The proposed descriptor is validated with the relative performance measures and is given in the sub section III-B in the wake of the other reported descriptors.

\section{A. Processing of CPF $+G D$ Descriptor}

The present paper computes the proposed CPF signature given in Equation (1) with the log polar transformation of the input object and the power $\mathrm{n}$ value as 2 . The only imaginary values of the corner potential flow are considered for constructing the shape signature. The proposed signature is applied on the objects of Kimia 99 database and the CPF signature of three Hand images (Hand1, Hand5 and Hand11) are illustrated in Figure 2. It is observed that the CPF of different objects of the same group are found to be similar.

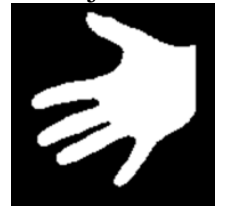

(i)

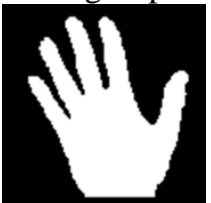

(ii)

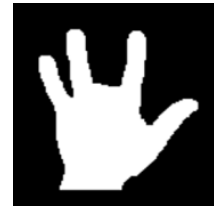

(iii)

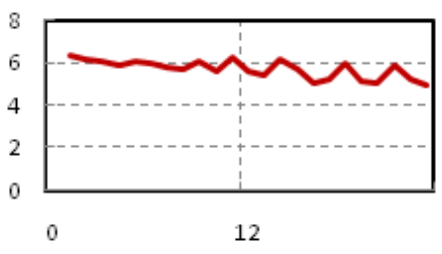

(iv)

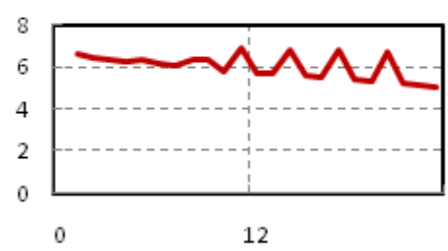

(v)

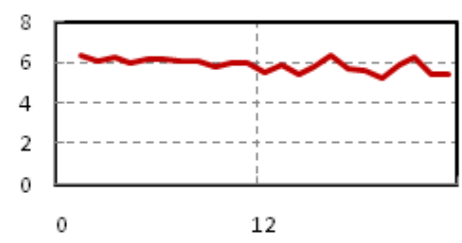

(vi)

Figure 2. (i) Hand1 Object (ii) Hand5 Object (iii) Hand11 Object (iv) CFP Signature of Hand1 (v) CFP Signature of Hand5 (vi) CFP Signature of Hand11.

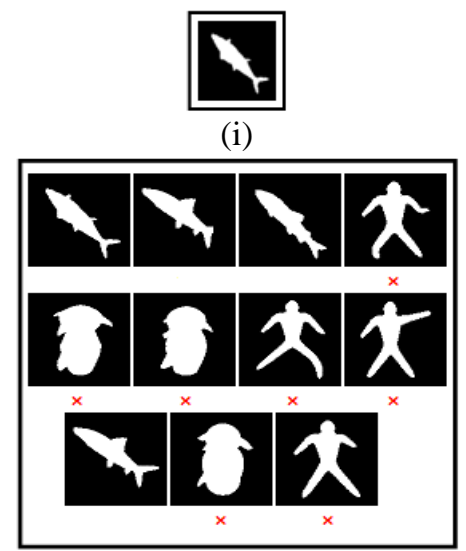

(ii)

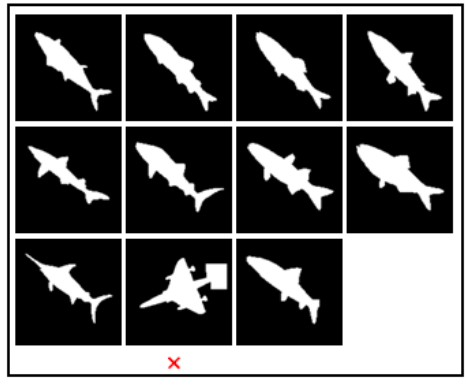

(iii)

Figure 3. (i) Fish4 Image (ii) ZMD Recognition Result of Fish4 (iii) CPF+GD Recognition Result of Fish4.

Next, the one dimensional Fourier transformation is applied on the designed CPF signature which yields invariant feature vector. Then, the Euclidean Distance (ED) is used for the shape toning process. This will compute the distance between test and target feature vectors and are allocated with the rank according to the distance. The performance of the proposed descriptor is estimated by the top eleven (groupsize) retrieved objects. Fig. 3 illustrates the recognition results of Fish4 object from Kimia 99 database. The Fig. 3(i) represents the input object, 3(ii) represents the ZMD recognition result and 3(iii) represents the $C P F+G D$ recognition result. From this, it is observed that the proposed $\mathrm{CPF}+\mathrm{GD}$ descriptor reduces the dissimilar recognition result than the ZMD.

\section{B. Validation of $C P F+G D$ descriptor}

With the computation of confusion matrix, the present paper performs the validation of the proposed CPF+GD descriptor. The present paper compares the precision of the proposed and four other standard descriptors viz., Zernike Moment Descriptor (ZMD), Angular Radial Transform Descriptor (ARTD), Moment Invariant Descriptor (MID) and Curvature Scale Space Descriptor (CSSD). The Table I gives the comparison of APLR and APHR measures of proposed and standard descriptors.

From Table I, it is observed that the CSSD descriptor is having lowest APLR and APHR values than other standard descriptors where as the ZMD is having highest APLR and APHR values. The proposed CPF+GD descriptor is found to be efficient and is giving improved results of both APLR and APHR than all the other methods. The PR plot [18] of all the five descriptors is shown in Figure 4. The CPF+GD 
descriptor clearly witnesses an improved precision prevalently at all recalls from 0 to 100 than the other descriptors. Among them, the CSSD descriptor is having low precision than the other descriptors.

TABLE I. APLR AND APHR OF PROPOSED DESCRIPTOR WITH KIMIA-99 DATABASE.

\begin{tabular}{|c|c|c|c|}
\hline Descriptor & APLR & APHR & $\begin{array}{c}\text { Average } \\
\text { Precision }\end{array}$ \\
\hline CPF+GD & 92.40 & 63.87 & 78.12 \\
\hline ARTD & 84.26 & 45.72 & 64.99 \\
\hline MID & 81.96 & 44.74 & 63.35 \\
\hline ZMD & 89.61 & 61.37 & 75.49 \\
\hline CSSD & 82.32 & 44.11 & 63.22 \\
\hline
\end{tabular}

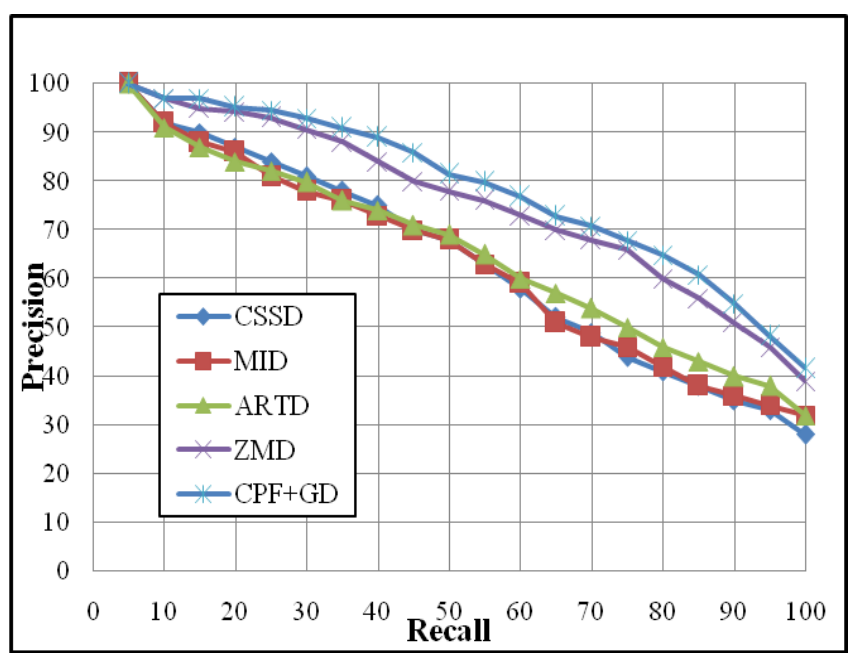

Figure 4. Comparison PR plot of $\mathrm{CPF}+\mathrm{GD}$ and other standard descriptors.

\section{Conclusions}

- Representation and description of shape of the object is found to be efficient with the complex potential.

- The contour through potential flow yields improved recognition results than the standard descriptors.

- The performance measures viz., TPR, SPC, PPV, FDR and ACC gives the detailed description of the efficiency of the proposed CPF+GD descriptor.

\section{References}

[1] Felzenszwalb P.F., Huttenlocher D., "Pictorial Structures for Object Recognition," International Journal of Computer Vision, Vol. 61, Issue.1, pp. 55-79, 2005.

[2] Borenstein E., Ullman S., "Class-specific, top-down segmentation," ECCV, vol. 2, pp. 109-122, 2002.

[3] Levin A., Weiss Y., "Learning to combine bottom-up and top-down segmentation," CCV, pp. 105-118, 2006.

[4] Leibe B., Seemann E., Schiele B., "Pedestrian detection in crowded scenes," CVPR, pp. 878-885, 2005.

[5] Ferrari V., Tuytelaars T., Gool L.J.V., "Object detection by contour segment networks," ECCV, pp. 14-28, 2006.
[6] Kokkinos I., Maragos P., Yuille A.L., "Bottom-up \& top-down object detection using primal sketch features and graphical models," CVPR, 2006.

[7] Zhao L., Davis L.S., "Closely coupled object detection and segmentation," ICCV, 2005.

[8] Ren X., Berg A.C., Malik J., "Recovering human body configurations using pairwise constraints between parts," ICCV, 2005.

[9] Mori G., Ren X., Efros A.A., Malik J., "Recovering human body configurations: Combining segmentation and recognition," CVPR, 2004.

[10] Srinivasan P., Shi J., "Bottom-up recognition and parsing of the human body," CVPR, 2007.

[11] Belongie S., Malik J., Puzicha J., "Shape matching and object recognition using shape contexts," IEEE Trans. Pattern Anal. Mach. Intell., Vol. 24, Issue. 4, 2002.

[12] Dalal N., Triggs B., "Histograms of oriented gradients for human detection," CVPR, 2005.

[13] Mani M.R., Varma G.P.S., Potukuchi D.M., Satyanarayana Ch., "A Modified Shape Context Method for Shape based Object Retrieval," SpringerPlus, Vol. 3, Issue. 1, pp. 1-12, 2014.

[14] Zhang D., Lu G., "Shape-based image retrieval using generic Fourier descriptor," Signal Processing: Image Communication, Vol. 17, pp. $825-848,2002$.

[15] Gope C., Kehtarnavaz N., "Affine invariant comparison of point-sets using convex hulls and hausdorff distances," Pattern Recognition, Vol. 40, Issue. 1, pp. 309-320, 2007.

[16] Goshtasby A., "Description and discrimination of planar shapes using shape matrices," IEEE Transactions on Pattern Analysis and Machine Intelligence, Vol. 7, pp. 738-743, 1985.

[17] Mani M.R., Varma G.P.S., Potukuchi D.M., Satyanarayana Ch., "Design of a Novel Shape Signature by Farthest Point Angle for Object Recognition," International Journal of Image, Graphics and Signal Processing, Vol.7, Issue. 1, pp. 35-46, 2015.

[18] Mani M.R., Varma G.P.S., Potukuchi D.M., Satyanarayana Ch., "Curvature for Fourier Heat Transformation Method: A Shape based Object Recognition," Studies in System Science (SSS), Vol. 2, pp. 9195, 2014.

[19] El Bashir T.B.A., "Flow Generated by a Vortex Outside A Circular Cylinder," International Mathematical Forum, Vol. 1, Issue. 21, pp. 1017-1032, 2006.

[20] Currie I.G., in "Fundamental Mechanics of Fluids," 4th Edition, CRC Press.

[21] Mani M.R., Varma G.P.S., Potukuchi D.M., Satyanarayana Ch., "A Conformal Mapping based Shape Signature for Object Recognition," WSEAS- $15^{\text {th }}$ International Conference on Applied Computer Science (ACS '15) May 20-22, 2015, Konya, Turkey.

[22] http://web.mit.edu/fluidsmodules/www/potential_flows/LecturesHTML/lec1011/node36.html 